\title{
Applications of Polyhedral Combinatorics to Multicommodity Flows and Compact Surfaces
}

\author{
A. SCHRIJVER
}

\begin{abstract}
We survey results on graphs and curves on compact surfaces and outline the proof methods, which make essential use of polyhedral combinatorics. We derive results on planar multicommodity flows.
\end{abstract}

\section{Introduction}

In this paper we give a survey of some recent results on multicommodity flows and compact surfaces, derived with the help of methods from polyhedral combinatorics. For several of the results obtained we know, at this moment, no other proof method than polyhedral methods.

In fact, these polyhedral methods are none other than two well-known variants of Farkas' lemma. Let $a_{1}, \ldots, a_{k}, b_{1}, \ldots, b_{m}$ be vectors in $\mathbf{R}^{n}$. The first variant is the "blocking polyhedron theorem" of Fulkerson [2]:

(1) if the polyhedron $\left\{x \in \mathbf{R}^{n} \mid x \geq 0 ; a_{i}^{\mathrm{T}} x \geq 1(i=1, \ldots, k)\right\}$ has vertices $b_{1}, \ldots, b_{m}$, then the polyhedron $\left\{x \in \mathbf{R}^{n} \mid x \geq\right.$ $\left.0 ; b_{j}^{\mathrm{T}} x \geq 1(j=1, \ldots, m)\right\}$ has all its vertices among $a_{1}$, $\ldots, a_{k}$

(assuming $a_{1}, \ldots, a_{k} \geq 0$ ).

The second variant is the "cone-form" of Farkas' lemma:

(2) if the convex cone $\left\{x \in \mathbf{R}^{n} \mid a_{i}^{\mathrm{T}} x \geq 0 \quad(i=1, \ldots, k)\right\}$ is generated by $b_{1}, \ldots, b_{m}$, then the convex cone $\left\{x \in \mathbf{R}^{n} \mid b_{j}^{\mathrm{T}} x \geq\right.$ $0(j=1, \ldots, m)\}$ is generated by $a_{1}, \ldots, a_{k}$.

The first variant is applied to graphs embedded on the Klein bottle (Section 2), and the second variant is applied to graphs embedded on compact orientable surfaces (Section 3).

1980 Mathematics Subject Classification (1985 Revision). Primary 05C10, 05C38, 90B10, $90 C 27$.

Key words and phrases. Klein bottle, compact surface, multicommodity flow, graph, planar, homotopy. 


\section{The Klein bottle}

We first focus on the Klein bottle and its relations to planar multicommodity flows. The Klein bottle is a compact surface usually represented as follows. Consider an annulus (= cylinder) and identify the inner and outer boundaries, in opposite orientation. Schematically:

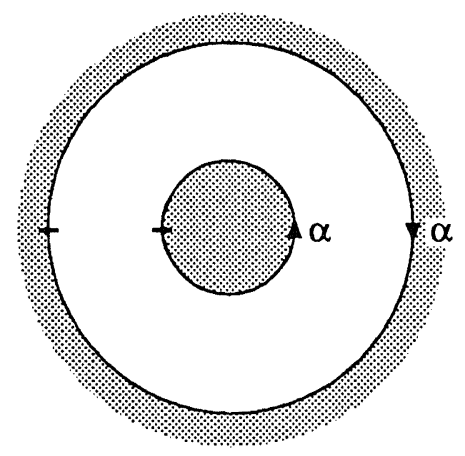

There is an alternative way of obtaining the Klein bottle from the annulus: identify opposite points on the outer boundary, and similarly, identify opposite points on the inner boundary. Schematically:

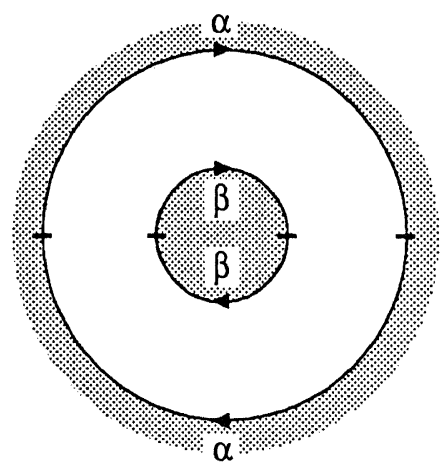

This corresponds to representing the Klein bottle as a 2-dimensional sphere with two "cross-caps" (one made by the outer boundary in (4), the other by the inner boundary).

The Klein bottle is a nonorientable surface. Hence there are two types of closed curves on the Klein bottle:

- orientation-preserving closed curves: those where the meaning of left and right is unchanged after one turn;

- orientation-reversing closed curves: those where the meaning of left and right is flipped after one turn.

It is not difficult to see that a closed curve is orientation-preserving (orientation-reversing, respectively) if it traverses the cross-caps an even (odd, respectively) number of times. 
Now let $G=(V, E)$ be a graph embedded on the Klein bottle $S$. (By a graph we mean an undirected graph. Embedding assumes nonintersecting edges except for their end vertices. Cellularly embedding means that all faces are homeomorphic to open disks. We identify an embedded graph with its image.)

We will be interested in the orientation-reversing circuits in $G$. (A circuit is a simple closed curve in $G$. We identify a circuit with the set of edges traversed by it.)

Call a set $B$ of edges a blocker if it intersects each orientation-reversing circuit. In [10] we proved the following min-max relations:

(i) If $G$ is bipartite, then the minimum size of an orientation-reversing circuit is equal to the maximum of pairwise disjoint blockers.

(ii) If $G$ is Eulerian, then the minimum size of a blocker is equal to the maximum number of pairwise edgedisjoint orientation-reversing circuits.

(Here a graph is Eulerian if all degrees are even.)

We here sketch a proof of these equalities. In fact, we first show (6)(i), and next derive (ii) from (i) with the blocking polyhedron variant (1) of Farkas' lemma. [7]:

The starting point in the proof method is the following result proved in

THEOREM 1. Let $G=(V, E)$ be a planar bipartite graph embedded in the plane. Let $I_{1}$ and $I_{2}$ be two of its faces. Then there exist pairwise edge-disjoint cuts $\delta\left(X_{1}\right), \ldots, \delta\left(X_{t}\right)$ so that for each two vertices $v, w$ with $v, w \in \operatorname{bd}\left(I_{1}\right)$ or $v, w \in \operatorname{bd}\left(I_{2}\right)$, the distance in $G$ from $v$ to $w$ is equal to the number of cuts $\delta\left(X_{j}\right)$ separating $v$ and $w$.

Here $\delta(X)$ denotes the set of nonloop edges of $G$ with exactly one end point in $X$. The cut $\delta(X)$ is said to separate $v$ and $w$ if $v \neq w$ and $|\{v, w\} \cap X|=1$. By bd(..) we denote the boundary of .. . Faces are considered as open regions.

From Theorem 1 we derive (6)(i):

THEOREM 2. Let $G=(V, E)$ be a bipartite graph embedded on the Klein bottle $S$. Then the minimum length of any orientation-reversing circuit in $G$ is equal to the maximum number of pairwise disjoint blockers.

Proof. Clearly, the maximum is not larger than the minimum. To show equality, we may assume that each face of $G$ is orientable, i.e., contains no cross-cap. Indeed, if a face contains a cross-cap, we can add a path to $G$ over this cross-cap in such a way that the graph remains bipartite and such that the minimum length of any orientation-reversing circuit remains unchanged (by taking the path with length large enough and of appropriate parity). 
Let $C_{1}$ be a minimum-length orientation-reversing circuit in $G$, say with length $t_{1}$. We "cut open" the Klein bottle $S$ along $C_{1}$. In this way we obtain a bordered surface $S^{\prime}$, with a circle $B_{1}$ as border, so that $S$ arises from $S^{\prime}$ by identifying opposite points on $B_{1}$. So $S^{\prime}$ is a Möbius strip. Let $i: S^{\prime} \rightarrow S$ denote the identification map. The graph $G^{\prime}:=i^{-1}[G]$ is a bipartite graph on $S^{\prime}$, and $B_{1}=i^{-1}\left[C_{1}\right]$.

Let $C_{2}$ be a minimum-length orientation-reversing circuit in $G^{\prime}$ (on $S^{\prime}$ ), say with length $t_{2}$. We may assume that $C_{2}$ is edge-disjoint from $B_{1}$ (by adding parallel edges). Next we "cut open" the Möbius strip $S^{\prime}$ along $C_{2}$. We now obtain an annulus $S^{\prime \prime}$, with two circles $B_{1}$ and $B_{2}$ as boundaries (in the ideal case where $C_{2}$ is vertex-disjoint from $B_{1}$-the general case is similar).

The Klein bottle $S$ arises from the annulus $S^{\prime \prime}$ by identifying opposite points on $B_{1}$ and by identifying opposite points on $B_{2}$. Let $i^{\prime}: S^{\prime \prime} \rightarrow S$ be the identification map, and let $G^{\prime \prime}:=\left(i^{\prime}\right)^{-1}[G]$. So $G^{\prime \prime}$ is a planar bipartite graph, embeddable in the plane $\mathbf{R}^{2}$, in such a way that two of its faces $I_{1}$ (= unbounded face) and $I_{2}$ have the following properties:

(i) the boundary of $I_{1}$ is a circuit $B_{1}$ of length $2 t_{1}$, and the boundary of $I_{2}$ is a circuit $B_{2}$ of length $2 t_{2}$;

(ii) $S$ arises from $\mathbf{R}^{2} \backslash\left(I_{1} \cup I_{2}\right)$ by identifying pairs of opposite points on $B_{1}$ and by identifying pairs of opposite points on $B_{2}$.

In fact, we identify $S^{\prime \prime}$ and $\mathbf{R}^{2} \backslash\left(I_{1} \cup I_{2}\right)$.

Since $t_{1}$ is the minimum length of an orientation-reversing circuit in $G$, each pair of opposite vertices on $B_{1}$ has distance exactly $t_{1}$. Similarly, since $t_{2}$ is the minimum length of an orientation-reversing circuit in $G^{\prime}$, each pair of opposite vertices on $B_{2}$ has distance exactly $t_{2}$.

By Theorem 1, there exist pairwise disjoint cuts $\delta\left(X_{1}\right), \ldots, \delta\left(X_{t}\right)$ so that for each two vertices $v$ and $w$ of $G^{\prime \prime}$ with $v, w \in \operatorname{bd}\left(I_{1}\right)$ or $v, w \in \operatorname{bd}\left(I_{2}\right)$, the distance in $G^{\prime \prime}$ from $v$ to $w$ is equal to the number of cuts $\delta\left(X_{j}\right)$ separating $v$ and $w$. We may assume that each $\delta\left(X_{j}\right)$ separates at least one such pair $v, w$ (all other cuts can be deleted).

Each cut $\delta\left(X_{j}\right)$ intersects any subpath $P$ of $B_{1}$ of length $t_{1}$ at most once (as $P$ is intersected by $t_{1}$ of the $\delta\left(X_{j}\right)$, as $P$ is a shortest path between its end points). So if $\delta\left(X_{j}\right)$ intersects $B_{1}$, it intersects $B_{1}$ exactly twice, in two opposite edges. Similarly, if $\delta\left(X_{j}\right)$ intersects $B_{2}$, it intersects $B_{2}$ exactly twice, in two opposite edges. 
We classify $\delta\left(X_{1}\right), \ldots, \delta\left(X_{t}\right)$ into three classes:

(i) those intersecting both $B_{1}$ and $B_{2}$, say $\delta\left(X_{1}\right), \ldots, \delta\left(X_{s}\right)$;

(ii) those intersecting $B_{1}$ but not $B_{2}$, say $\delta\left(X_{s+1}\right), \ldots, \delta\left(X_{t_{1}}\right)$;

(iii) those intersecting $B_{2}$ but not $B_{1}$, say $\delta\left(X_{t_{1}+1}\right), \ldots, \delta\left(X_{t}\right)$.

Note that $B_{2}$ is intersected by exactly $t_{2}$ of the $\delta\left(X_{j}\right)$, and hence $t_{2}=$ $s+\left(t-t_{1}\right)$, i.e., $s=t_{1}+t_{2}-t$.

Now it is not difficult to see that the images of the $\delta\left(X_{j}\right)$, properly composed, give blockers in $G$ as required. In fact, we can take:

(9)

$i^{\prime}\left[\delta\left(X_{1}\right)\right], \ldots, i^{\prime}\left[\delta\left(X_{s}\right)\right], i^{\prime}\left[\delta\left(X_{s+1}\right) \cup \delta\left(X_{t_{1}+1}\right)\right], \ldots, i^{\prime}\left[\delta\left(X_{t_{1}}\right) \cup \delta\left(X_{2 t_{1}-s}\right)\right]$

A standard corollary in polyhedral combinatorics now is:

Theorem 3. Let $G=(V, E)$ be a graph embedded on the Klein bottle $S$. Then each vertex of the polytope in $\mathbf{R}^{E}$ determined by

$$
\begin{aligned}
& \text { (i) } x(e) \geq 0 \quad(e \in E), \\
& \text { (ii) } \sum_{e \in C} x(e) \geq 1 \quad(C \text { orientation-reversing circuit })
\end{aligned}
$$

is the incidence vector of some blocker.

Proof. Let $x$ be a positive rational vector satisfying (10). We show that there exist blockers $B_{1}, \ldots, B_{t}$ and rationals $\lambda_{1}, \ldots, \lambda_{t}>0$ so that $\lambda_{1}+$ $\cdots+\lambda_{t}=1$ and so that

$$
x \geq \lambda_{1} \chi^{B_{1}}+\cdots+\lambda_{t} \chi^{B_{1}}
$$

(where $\chi^{B}$ denotes the incidence vector of $B$ ). This suffices to prove the theorem.

Let $N$ be a natural number so that $N x(e)$ is an even integer for each edge $e$. Replace each edge of $G$ by a path of length $N x(e)$ (that is, put $N x(e)-1$ new vertices on $e$ ). We obtain a bipartite graph $G^{\prime}$. Let $C^{\prime}$ be a minimum-length orientation-reversing circuit in $G^{\prime}$, of length $t$, say. As $x$ satisfies (10), we know $t \geq N$. By Theorem 2 there exist $t$ pairwise edge-disjoint blockers $B_{1}^{\prime}, \ldots, B_{t}^{\prime}$ in $G^{\prime}$. Their "projections" to $G$ give $t$ blockers $B_{1}, \ldots, B_{t}$ in $G$ with the property that each edge $e$ of $G$ is contained in at most $N x(e)$ of the $B_{j}$. Hence

$$
t x \geq N x \geq \chi^{B_{1}}+\cdots+\chi^{B_{1}} .
$$

Taking $\lambda_{j}:=1 / t$ for each $j$ gives $(11)$. 
Lehman's theorem (1) now implies the dual statement of Theorem 3:

THEOREM 4. Let $G=(V, E)$ be a graph embedded on the Klein bottle $S$. Then the vertices of the polytope in $\mathbf{R}^{E}$ determined by

(i) $x(e) \geq 0 \quad(e \in E)$,

(ii) $\sum_{e \in B} x(e) \geq 1 \quad(B \subseteq E, B$ blocker $)$

are exactly the characteristic vectors of orientation-reversing circuits.

This is in fact the fractional packing version of (6)(ii). We derive the integer packing result (6)(ii):

THEOREM 5. Let $G=(V, E)$ be an Eulerian graph embedded on the Klein bottle $S$. Then the minimum size of a blocker is equal to the maximum number of pairwise edge-disjoint orientation-reversing circuits.

Proof. Clearly, the maximum is not more than the minimum. Suppose equality does not hold, and let $G$ form a counterexample with

$$
\sum_{v \in V} 2^{\operatorname{deg}(v)}
$$

as small as possible (where $\operatorname{deg}(v)$ denotes the degree of $v$ ). Then

$$
\text { each vertex of } G \text { has degree at most } 4 \text {. }
$$

For suppose $v$ has degree at least 6 :

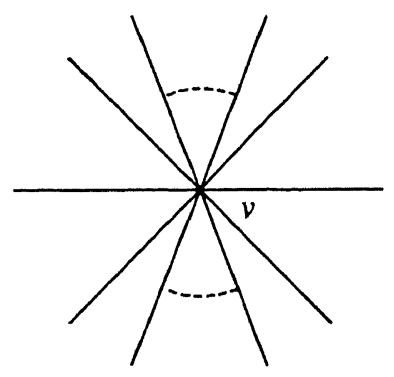

Replace this (on the Klein bottle) by:

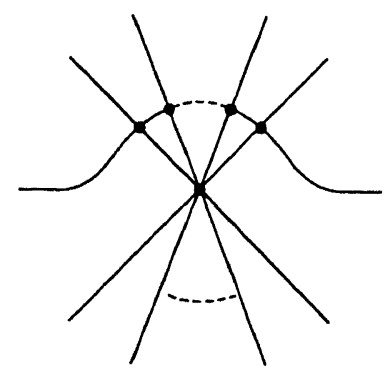

This modification does not change the minimum size, $t$ say, of a blocker, as one may check. However, it reduces the sum (14), so in the new graph there exist $t$ pairwise edge-disjoint orientation-reversing circuits. This gives also 
in the original graph $t$ pairwise edge-disjoint orientation-reversing circuits, contradicting our assumption.

This shows (15). Let $t$ be the minimum size of a blocker in $G$. Hence the vector $x$ with all entries equal to $1 / t$ satisfies (13). So by Theorem 4 there exist orientation-reversing circuits $C_{1}, \ldots, C_{k}$ (pairwise different) and reals $\lambda_{1}, \ldots, \lambda_{k}>0$ so that

$$
\begin{aligned}
& \text { (i) } \lambda_{1}+\cdots+\lambda_{k}=1 \\
& \text { (ii) } \lambda_{1} \chi^{C_{1}}+\cdots+\lambda_{k} \chi^{C_{k}} \leq x \text {. }
\end{aligned}
$$

Consider a vertex $v$ of $G$ of degree 4 , and the edges $e_{1}, e_{2}, e_{3}, e_{4}$ incident to $v$ in cyclic order:

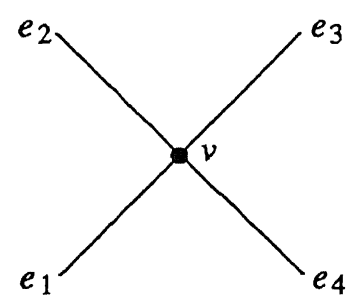

Thus $e_{1}$ and $e_{3}$ are "opposite" in $v$, and similarly, $e_{2}$ and $e_{4}$ are opposite in $v$. We show that for each circuit $C_{i}$

$$
\begin{aligned}
& \text { (i) } C_{i} \text { traverses } e_{1} \Longleftrightarrow C_{i} \text { traverses } e_{3} \text {, } \\
& \text { (ii) } C_{i} \text { traverses } e_{2} \Longleftrightarrow C_{i} \text { traverses } e_{4} \text {. }
\end{aligned}
$$

Having shown this for each vertex $v$ and each $C_{i}$, it follows that $C_{1}, \ldots, C_{k}$ are pairwise edge-disjoint. Since $k \geq t$ (since $\lambda_{i} \leq 1 / t$ for each $i$ ), this proves the theorem.

If (20) does not hold, we may assume without loss of generality that $C_{1}$ traverses $e_{1}$ and $e_{2}$. Replace (19) by

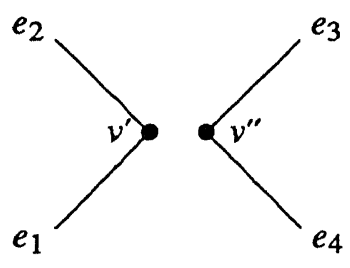

Let $G^{\prime}$ be the new graph obtained. So $G$ arises from $G^{\prime}$ by identifying $v^{\prime}$ and $v^{\prime \prime}$. Graph $G^{\prime}$ is Eulerian again, with sum (14) smaller than for $G$. So by the minimality assumption, the theorem to be proved holds for $G^{\prime}$.

Let $t^{\prime}$ be the minimum size of a blocker in $G^{\prime}$. If $t^{\prime} \geq t$, there exist $t$ pairwise edge-disjoint orientation-reversing circuits in $G^{\prime}$, and hence also in $G$, contradicting our assumption. So $t^{\prime}<t$. By the Euler condition, $t^{\prime} \leq t-2$. Let $B^{\prime}$ be a blocker of size $t^{\prime}$ in $G^{\prime}$. Then $B:=B^{\prime} \cup\left\{e_{1}, e_{2}\right\}$ is a blocker of size at most $t^{\prime}+2$ in $G$. Since $|B| \geq t$, we know $|B|=t$. 
Since $\left|C_{i} \cap B\right| \geq 1$ while $\left|C_{1} \cap B\right|>1$, this gives the contradiction:

$$
\begin{aligned}
1= & \sum_{e \in B} 1 / t \geq \sum_{e \in B}\left(\lambda_{1} \chi^{C_{1}}(e)+\cdots+\lambda_{k} \chi^{C_{k}}(e)\right) \\
= & \lambda_{1}\left|C_{1} \cap B\right|+\lambda_{2}\left|C_{2} \cap B\right|+\cdots+\lambda_{k}\left|C_{k} \cap B\right| \\
& >\lambda_{1}+\cdots+\lambda_{k}=1 .
\end{aligned}
$$

Theorem 5 has a number of corollaries. First, a theorem of Lins [3] follows, which is in fact the analogue of Theorem 5 for the projective plane. Note that the orientation-reversing circuits in the projective plane are exactly the non-null-homotopic circuits, and exactly the nonseparating circuits.

THEOREM 6 (Lins' theorem). Let $G=(V, E)$ be an Eulerian graph embedded in the projective plane. Then the maximum number of pairwise edgedisjoint non-null-homotopic circuits in $G$ is equal to the minimum number of edges intersected by any non-null-homotopic closed curve not intersecting $V$.

PRoof. This follows directly from Theorem 5 by putting a cross-cap in one of the faces of $G$, thus transforming the projective plane to a Klein bottle. Note that the minimal blockers in $G$ are exactly the minimal sets of edges intersected by some non-null-homotopic closed curve not intersecting $V$.

Theorem 5 also implies two results on planar multicommodity flows. Let $G=(V, E)$ be a graph, and let $r_{1}, \ldots, r_{k}, s_{1}, \ldots, s_{k}$ be vertices of $G$ (so that $r_{i} \neq s_{i}$ for all $i$ ). Clearly, the following cut condition is a necessary condition for the existence of pairwise edge-disjoint paths $P_{1}, \ldots, P_{k}$ where $P_{i}$ connects $r_{i}$ and $s_{i}(i=1, \ldots, k)$ :

(cut condition): for each $X \subseteq V:|\delta(X)| \geq$ number of pairs $r_{i}, s_{i}$ separated by $\delta(X)$.

Simple examples show that this cut condition is not sufficient in general.

However, an Euler condition turns out to be quite helpful:

$$
\begin{aligned}
& \text { (Euler condition) the graph }\left(V, E \cup\left\{\left\{r_{1}, s_{1}\right\}, \ldots,\left\{r_{k}, s_{k}\right\}\right\}\right) \\
& \text { is Eulerian. }
\end{aligned}
$$

First we sketch how to derive

Theorem 7. Let $G=(V, E)$ be a planar graph embedded in the plane $\mathbf{R}^{2}$ and let $r_{1}, \ldots, r_{k}, s_{1}, \ldots, s_{k}$ be vertices of $G$ so that the Euler condition holds. Let $r_{1}, \ldots, r_{k}$ be incident to the unbounded face $I_{1}$ in clockwise order. Let $s_{1}, \ldots, s_{k}$ be incident to some other face $I_{2}$ in counterclockwise order. Then there exist pairwise edge-disjoint paths $P_{1}, \ldots, P_{k}$ where $P_{i}$ connects $r_{i}$ and $s_{i}(i=1, \ldots, k)$ if and only if the cut condition is satisfied.

Proof. Let the cut condition be satisfied. Extend $\mathbf{R}^{2} \backslash\left(I_{1} \cup I_{2}\right)$ to the Klein bottle, by adding a cylinder between the boundaries of $I_{1}$ and $I_{2}$. Extend $G$ to a graph $G^{\prime}$ on the Klein bottle by adding edges $e_{1}, \ldots, e_{k}$ 
over this cylinder, in such a way that $e_{i}$ connects $r_{i}$ and $s_{i}(i=1, \ldots, k)$. Then a circuit in $G^{\prime}$ is orientation-reversing if and only if it contains an odd number of edges from $e_{1}, \ldots, e_{k}$. So it suffices to show that $G^{\prime}$ contains $k$ pairwise edge-disjoint orientation-reversing circuits.

Since $G^{\prime}$ is Eulerian, we can apply Theorem 5 . That is, we must show that each blocker in $G^{\prime}$ has size at least $k$. It is not difficult to derive this from the cut condition.

Also a theorem of Okamura [4] can be derived:

THEOREM 8 (Okamura's theorem). Let $G=(V, E)$ be a planar graph embedded in the plane $\mathbf{R}^{2}$ and let $r_{1}, \ldots, r_{k}, s_{1}, \ldots, s_{k}$ be vertices of $G$ so that the Euler condition holds. Let there exist two faces $I_{1}$ and $I_{2}$ of $G$ so that for each $i=1, \ldots, k, r_{i}, s_{i} \in \operatorname{bd}\left(I_{1}\right)$ or $r_{i}, s_{i} \in \operatorname{bd}\left(I_{2}\right)$. Then there exist pairwise edge-disjoint paths $P_{1}, \ldots, P_{k}$ where $P_{i}$ connects $r_{i}$ and $s_{i}(i=1, \ldots, k)$ if and only if the cut condition is satisfied.

PRoof. Without loss of generality, $I_{1}$ is the unbounded face, and $r_{1}, \ldots$, $r_{t}, s_{1}, \ldots, s_{t} \in \mathrm{bd}\left(I_{1}\right)$ and $r_{t+1}, \ldots, r_{k}, s_{t+1}, \ldots, s_{k} \in \mathrm{bd}\left(I_{2}\right)$. By an argument due to $\mathrm{S}$. Lins we may assume that $r_{1}, \ldots, r_{t}, s_{1}, \ldots, s_{t}$ occur in this order cyclically around $\operatorname{bd}\left(I_{1}\right)$. To see this, first note that we may assume that the vertices $r_{1}, \ldots, r_{t}, s_{1}, \ldots, s_{t}$ are distinct and have degree 1 (as we can add a new vertex of degree 1 to any $r_{i}$ or $s_{i}$ and replace this $r_{i}$ or $s_{i}$ by the new vertex). Call two pairs $r_{i}, s_{i}$ and $r_{j}, s_{j}$ on bd( $\left.I_{1}\right)$ crossing if $i \neq j$ and $r_{i}, r_{j}, s_{i}, s_{j}$ occur in this order cyclically around $\mathrm{bd}\left(I_{1}\right)$, clockwise or counterclockwise. Suppose not all pairs $r_{i}, s_{i}$ are crossing. Then there exist $i, j$ so that $r_{i}, s_{i}$ and $r_{j}, s_{j}$ are noncrossing and so that there is no pair $r_{h}, s_{h}$ on the part of the boundary of $I_{1}$ that connects $r_{i}$ and $s_{i}$ and that does not contain $s_{i}$ or $s_{j}$ (maybe after exchanging $r_{i}$ and $s_{i}$ ).

Now we can add in $I_{1}$ three new vertices, $w, r_{i}^{\prime}$, and $r_{j}^{\prime}$, and four new edges as follows:

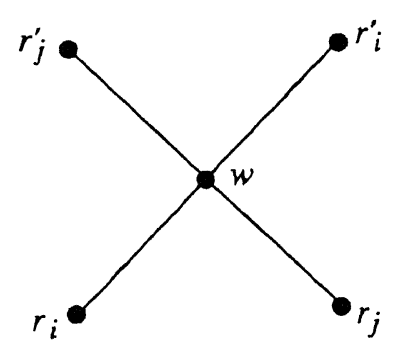

Replacing $r_{i}$ and $r_{j}$ by $r_{i}^{\prime}$ and $r_{j}^{\prime}$ does not violate the cut condition. Moreover, any pair of edge-disjoint paths $P_{i}^{\prime}, P_{j}^{\prime}$ in the extended graph, where $P_{i}^{\prime}$ connects $r_{i}^{\prime}$ and $s_{i}$ and $P_{j}^{\prime}$ connects $r_{j}^{\prime}$ and $s_{j}$, contains edge-disjoint paths $P_{i}$ and $P_{j}$ in the original graph, where $P_{i}$ connects $r_{i}$ and $s_{i}$ and $P_{j}$ connects $r_{j}$ and $s_{j}$.

Repeating this construction, we end up with $r_{1}, \ldots, r_{t}, s_{1}, \ldots, s_{t}$ occurring in this order cyclically around $\mathrm{bd}\left(I_{1}\right)$ (possibly after reordering indices 
and exchanging $r_{i}$ and $\left.s_{i}\right)$. Similarly, we can assume that $r_{t+1}, \ldots, r_{k}, s_{t+1}$, $\ldots, s_{k}$ occur in this order cyclically around bd $\left(I_{2}\right)$.

Now extend $\mathbf{R}^{2} \backslash\left(I_{1} \cup I_{2}\right)$ to the Klein bottle, by adding two cross-caps (in fact, two Möbius strips) along the boundaries of $I_{1}$ and of $I_{2}$. Extend $G$ to a graph $G^{\prime}$ on the Klein bottle by adding edges $e_{1}, \ldots, e_{k}$ over the cross-caps, in such a way that $e_{i}$ connects $r_{i}$ and $s_{i}(i=1, \ldots, k)$. The remainder of the proof is similar to that of Theorem 7.

Okamura's theorem has as special case the theorem of Okamura and Seymour [5], where $r_{1}, \ldots, r_{k}, s_{1}, \ldots, s_{k}$ are all on the boundary of one face.

\section{Compact orientable surfaces}

We next show how some results on curves and graphs on compact orientable surfaces can be derived with the help of polyhedral combinatorics. Recall that a compact orientable surface is a 2-dimensional sphere with a finite number of "handles" added.

Let $S$ be a compact orientable surface. A closed curve on $S$ is a continuous function $C: S_{1} \rightarrow S$, where $S_{1}$ is the unit circle. We call two closed curves $C$ and $C^{\prime}$ homotopic, in notation: $C \sim C^{\prime}$, if $C$ can be shifted continuously to $C^{\prime}$, without fixing a base point; in other words, there exists a continuous function $\Phi:[0,1] \times S_{1} \rightarrow S$ so that

$$
\Phi(0, z)=C(z) \text { and } \Phi(1, z)=C^{\prime}(z) \quad \text { for all } z \in S_{1} .
$$

We call a closed curve primitive if there do not exist a closed curve $D$ and an integer $n \geq 2$ so that $C \sim D^{n}$.

By $\operatorname{cr}(C, D)$ we denote the number of intersections of $C$ and $D$ (counting multiplicities):

$$
\operatorname{cr}(C, D):=\left|\left\{(y, z) \in S_{1} \times S_{1} \mid C(y)=D(z)\right\}\right| .
$$

By $\min \operatorname{cr}(C, D)$ we denote the minimum number of intersections of $C^{\prime}$ and $D^{\prime}$, ranging over all $C^{\prime} \sim C$ and $D^{\prime} \sim D$ :

$$
\min \operatorname{cr}(C, D):=\min \left\{\operatorname{cr}\left(C^{\prime}, D^{\prime}\right) \mid C^{\prime} \sim C, D^{\prime} \sim D\right\} .
$$

One objective in this section is to derive the following result in combinatorial topology [8]. It describes under which conditions two systems of primitive closed curves are homotopically the same:

THEOREM 17. Let $C_{1}, \ldots, C_{k}$ and $C_{1}^{\prime}, \ldots, C_{k^{\prime}}^{\prime}$ be primitive closed curves on $S$. Then the following are equivalent:

(i) $k=k^{\prime}$ and there exists a permutation $\pi$ of $\{1, \ldots, k\}$ so that $C_{\pi(i)}^{\prime} \sim C_{i}$ or $C_{\pi(i)}^{\prime} \sim C_{i}^{-1}$ for each $i=1, \ldots, k$;

(ii) for each closed curve $D$ on $S$ :

$$
\sum_{i=1}^{k} \min \operatorname{cr}\left(C_{i}, D\right)=\sum_{i=1}^{k^{\prime}} \min \operatorname{cr}\left(C_{i}^{\prime}, D\right) .
$$


The implication (ii) $\Rightarrow$ (i) is the essence of the theorem. It asserts that if two systems of primitive closed curves cannot be shifted to each other, then there exists a closed curve $D$ distinguishing between them. Note that we cannot skip the primitiveness condition.

A second objective is a result in topological graph theory [11]. We need some further terminology and notation. If $G$ is a graph embedded on $S$ and $D$ is a closed curve on $S$, we denote by $\operatorname{cr}(G, D)$ the number of intersections of $G$ and $D$ (counting multiplicities):

$$
\operatorname{cr}(G, D):=\left|\left\{z \in S_{1} \mid D(z) \in G\right\}\right| .
$$

By $\mu_{G}(D)$ we denote the minimum number of intersections of $G$ and $D^{\prime}$, ranging over all $D^{\prime} \sim D$ :

$$
\mu_{G}(D):=\min \left\{\operatorname{cr}\left(G, D^{\prime}\right) \mid D^{\prime} \sim D\right\} .
$$

If $G^{\prime}$ arises from $G$ by deleting edges and isolated vertices and by contracting nonloop edges, we say that $G^{\prime}$ is a minor of $G$. It is called a proper minor if at least one edge is deleted or contracted. Note that if $G^{\prime}$ is a minor of $G$ then $\mu_{G^{\prime}} \leq \mu_{G}$. We call $G$ a kernel (on $S$ ) if for each proper minor $G^{\prime}$ of $G$ one has $\mu_{G^{\prime}} \neq \mu_{G}$ (i.e., $\mu_{G^{\prime}}(D)<\mu_{G}(D)$ for at least one $D$ ).

The theorem states that a kernel $G$ is in a sense determined by $\mu_{G}$ :

THEOREM 18. Let $G$ and $G^{\prime}$ be cellularly embedded kernels on $S$ with $\mu_{G}=\mu_{G^{\prime}}$. Then $G^{\prime}$ can be obtained from $G$ by a series of the following operations:

(i) shifting the graph homotopically over $S$;

(ii) taking the (surface) dual graph;

(iii) $\Delta Y$-exchange.

Here we take the dual graph only if the graph is cellularly embedded on $S$ (i.e., every face is a disk). $\Delta Y$-exchange means replacing a triangular face by a new vertex of degree three, connected by edges to the three vertices of the triangle:
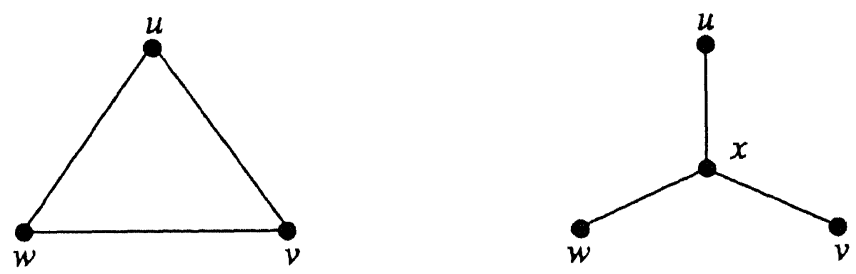

or conversely.

Note that each of the operations (i), (ii), (iii) keeps the function $\mu_{G}$ invariant. For the projective plane the analogue of Theorem 18 was proved by Scott Roundby [6].

We sketch how Theorems 17 and 18 are proved with the help of polyhedral results. The basic notion is that of a tight graph on $S$. For any graph 
$G=(V, E)$ on $S$ and any closed curve $D$ on $S$, let $\bar{\mu}_{G}(D)$ denote the minimum number of intersections of $G$ and $D^{\prime}$, ranging over all $D^{\prime} \sim D$ not intersecting $V$ :

$$
\bar{\mu}_{G}(D):=\min \left\{\operatorname{cr}\left(G, D^{\prime}\right) \mid D^{\prime} \sim D, D^{\prime} \text { does not intersect } V\right\} .
$$

If $G$ is 4-regular and $v$ is a vertex of $G$, we call replacing

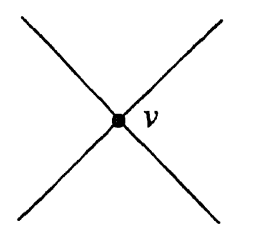

by

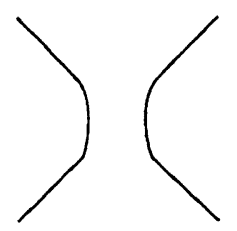

opening of $G$ at $v$ (there are two possible openings at $v$ ). If $G^{\prime}$ arises from $G$ by a series of openings, we call $G^{\prime}$ an opening of $G$. If there is at least one opening, it is called a proper opening.

Note that if $G^{\prime}$ is an opening of $G$ then $\bar{\mu}_{G^{\prime}} \leq \bar{\mu}_{G}$. We call $G$ tight (on $S$ ) if for each proper opening $G^{\prime}$ of $G$ one has $\bar{\mu}_{G^{\prime}} \neq \bar{\mu}_{G}$ (i.e., $\bar{\mu}_{G^{\prime}}(D)<\bar{\mu}_{G}(D)$ for at least one $D$ ).

If $G$ is a 4-regular graph on $S$, the straight decomposition of $G$ is the partition of the edges of $G$ into closed curves obtained as follows. Follow an edge, $e$ say, until one of its end points, $v$ say. Next continue along the edge, $e^{\prime}$ say, opposite in $v$ to $e$ :

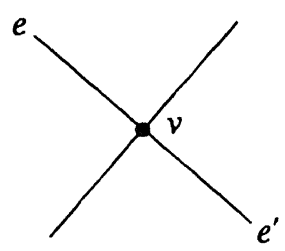

Similarly, if we arrive in the other end point of $e^{\prime}, v^{\prime}$ say, we continue along the edge opposite to $e^{\prime}$ in $v^{\prime}$. Repeating this, we finally will return in $e$. Thus we have obtained a closed curve.

Repeating this for the edges left, we obtain a system of closed curves $C_{1}, \ldots, C_{k}$ traversing each edge exactly once. Clearly, this system is unique up to the choice of the starting points of the curves and up to reversing any of the closed curves. We call $C_{1}, \ldots, C_{k}$ the straight decomposition of $G$.

In [9] we proved the following theorem:

THEOREM 9. Let $G$ be a 4-regular graph embedded on the compact orientable surface $S$. Then $G$ is tight if and only if the straight decomposition $C_{1}, \ldots, C_{k}$ forms a minimally crossing system of primitive closed curves.

Here $C_{1}, \ldots, C_{k}$ is called minimally crossing if any $C_{i}$ has a minimum number of self-crossings (over all $C_{i}^{\prime} \sim C_{i}$ ), and any two $C_{i}$ and $C_{j}$ have a minimum number of mutual crossings (over all $C_{i}^{\prime} \sim C_{i}$ and $C_{j}^{\prime} \sim C_{j}$ ).

Our proof in [9] is quite hard. From Theorem 9 one can derive quite easily 
(see [9]):

THEOREM 10. Let $G$ be a tight graph on the compact orientable surface $S$, with straight decomposition $C_{1}, \ldots, C_{k}$. Then for each closed curve $D$ on $S$ :

$$
\bar{\mu}_{G}(D)=\sum_{i=1}^{k} \min \operatorname{cr}\left(C_{i}, D\right) .
$$

We show that this implies

THEOREM 11. Let $G$ be an Eulerian graph embedded on the compact orientable surface $S$. Then the edges of $G$ can be partitioned into closed curves $C_{1}, \ldots, C_{k}$ in such a way that for each closed curve $D$ on $S$

$$
\bar{\mu}_{G}(D)=\sum_{i=1}^{k} \min \operatorname{cr}\left(C_{i}, D\right) .
$$

Proof. By applying the same modification as given by (16) and (17), we may assume that $G$ is 4-regular. Moreover, we may assume that $G$ is tight, as we can open $G$ at vertices as long as we do not change the function $\bar{\mu}_{G}$. Hence the theorem follows from Theorem 10.

The analogue of Theorem 11 for the projective plane is Lins' theorem (Theorem 6 ot above). At the moment we do not know a similar result for arbitrary compact nonorientable surfaces.

By passing to the surface dual graph, Theorem 11 transforms to

THEOREM 12. Let $G=(V, E)$ be a cellularly embedded bipartite graph on the compact orientable surface $S$. Then there exist closed curves $D_{1}, \ldots, D_{t}$ not intersecting $V$ and crossing (altogether) each edge exactly once, in such a way that for each closed curve $C$ on $S$ there exists a closed curve $C^{\prime} \sim C$ in $G$ with the property

$$
\text { length }_{G}\left(C^{\prime}\right)=\sum_{j=1}^{t} \min \operatorname{cr}\left(C, D_{j}\right) .
$$

[Here length ${ }_{G}\left(C^{\prime}\right)$ is the number of edges of $G$ traversed by $C^{\prime}$ (counting multiplicities).]

PROOF. The theorem follows directly by applying Theorem 11 to the surface dual graph of $G$.

It should be noted here that the theorem is not true if we delete "cellularly embedded."

Observe the analogy of Theorem 12 with Theorem 2 on the Klein bottle. We can now derive theorems analogous to Theorems 3 and 4, using the cone version (2) of Farkas' lemma. In fact, we only give the analogue of Theorem 4.

Let $G=(V, E)$ be a graph embedded on the compact orientable surface and let $C_{1}, \ldots, C_{k}$ be closed curves on $S$. Consider the convex cone $K$ in 
$\mathbf{R}^{k} \times \mathbf{R}^{E}$ generated by the vectors

$$
\begin{aligned}
& \text { (i) }\left(\varepsilon_{i} ; \chi^{C}\right)(i=1, \ldots, k ; C \text { closed curve in } G \text { with } \\
&\left.C \sim C_{1}\right) ; \\
& \text { (ii) }\left(0 ; \varepsilon_{e}\right) \quad(e \in E) .
\end{aligned}
$$

Here $\chi^{C}$ is the vector in $\mathbf{R}^{E}$ defined by

$$
\chi^{C}(e):=\text { number of times } C \text { traverses } e
$$

for $e \in E$. Moreover, $\varepsilon_{i}$ and $\varepsilon_{e}$ denote the $i$ th and $e$ th unit basis vectors in $\mathbf{R}^{k}$ and $\mathbf{R}^{E}$, respectively, while $\mathbf{0}$ is the all-zero vector in $\mathbf{R}^{k}$.

The cone $K$ is a polyhedral cone, i.e., is generated by only finitely many vectors among (38). To see this, observe that for each fixed $i=1, \ldots, k$, there exist only finitely many minimal vectors in the collection $\left\{\chi^{C} \mid C\right.$ is a closed curve in $G$ with $C \sim C_{i}$ \} (minimal with respect to componentwise comparison). This follows from the fact that these are vectors in $\mathbf{Z}_{+}^{E}$. We can restrict (38)(i) to those with $\chi^{C}$ such a minimal vector.

Now the analogue of Theorem 4 is

THEOREM 13. $K$ is exactly the set of vectors $(z ; x)$ in $\mathbf{R}^{k} \times \mathbf{R}^{E}$ satisfying

$$
\begin{array}{ll}
\text { (40) (i) } x(e) \geq 0 & (e \in E), \\
\text { (ii) } \sum_{e \in E} \gamma^{D}(e) \cdot x(e) \geq \sum_{i=1}^{k} \min \operatorname{cr}\left(C_{i}, D\right) \cdot z_{i} & \begin{array}{l}
\text { (D closed curve in } S \text { not } \\
\text { intersecting } V) .
\end{array}
\end{array}
$$

[Here $\gamma^{D}(e)$ denotes the number of times $D$ intersects $e$.]

Proof. It is not difficult to check that each vector (38) satisfies (40). Suppose next that some vector $(z ; x) \in \mathbf{R}^{k} \times \mathbf{R}^{E}$ satisfies (40) but does not belong to $K$. Then by Farkas' lemma (cone-form) there exists a vector $(p ; \ell)$ in $\mathbf{R}^{k} \times \mathbf{R}^{E}$ so that $(p ; \ell)$ has nonnegative inner product with all vectors (38) but not with $(z ; x)$. That is,

$$
\begin{array}{ll}
\text { (i) } p_{i}+\sum_{e \in E} \ell(e) \cdot \chi^{C}(e) \geq 0 & (i=1, \ldots, k ; C \text { closed } \\
\text { curve in } \left.G \text { with } C \sim C_{i}\right) & (e \in E) ; \\
\text { (ii) } \ell(e) \geq 0 & \\
\text { (iii) } \sum_{i=1}^{k} p_{i} z_{i}+\sum_{e \in E} \ell(e) x(e)<0 . &
\end{array}
$$

We may assume (by increasing $\ell(e)$ slightly) that $\ell$ is rational and positive. Hence we may assume (by blowing up $(p ; \ell)$ ) that each $\ell(e)$ is a positive even integer.

Now replace each edge $e$ of $G$ by a path of length $\ell(e)$ making the graph $G^{\prime}$. So $G^{\prime}$ arises from $G$ by putting $\ell(e)-1$ new vertices on any edge $e$. 
Moreover, we make $G^{\prime}$ cellularly embedded by adding paths over nondisk faces of length $T$, where $T$ is even and $T \geq \max \left\{-p_{1}, \ldots,-p_{k}\right\}$.

Since $C^{\prime}$ is bipartite, by Theorem 12 there exist closed curves $D_{1}, \ldots, D_{t}$ not intersecting the vertex set of $G^{\prime}$ and crossing each edge of $G^{\prime}$ exactly once, in such a way that for each $i=1, \ldots, k$ there exists a closed curve $C_{i}^{\prime} \sim C_{i}$ in $G^{\prime}$ with the property that

$$
\text { length }_{G^{\prime}}\left(C_{i}^{\prime}\right)=\sum_{j=1}^{t} \min \operatorname{cr}\left(C_{i}, D_{j}\right) .
$$

Note that

$$
\text { length }_{G^{\prime}}\left(C_{i}^{\prime}\right)=\sum_{e \in E} \ell(e) \chi^{C_{i}^{\prime}}(e) \quad \text { and } \ell(e)=\sum_{j=1}^{t} \gamma^{D_{j}}(e) .
$$

In particular, by $(41)(\mathbf{i})$

$$
p_{i}+\text { length }_{G^{\prime}}\left(C_{i}^{\prime}\right) \geq 0 \quad(i=1, \ldots, k) .
$$

This implies the following contradiction to (41)(iii):

$$
\begin{aligned}
&-\sum_{i=1}^{k} p_{i} z_{i} \leq \sum_{i=1}^{k} \text { length }_{G^{\prime}}\left(C_{i}^{\prime}\right) z_{i}=\sum_{i=1}^{k} \sum_{j=1}^{t} \min \operatorname{cr}\left(C_{i}, D_{j}\right) z_{i} \\
& \leq \sum_{j=1}^{t} \sum_{e \in E} \gamma^{D_{j}}(e) x(e)=\sum_{e \in E} \ell(e) x(e) .
\end{aligned}
$$

Theorem 13 implies the following "homotopic circulation theorem":

THEOREM 14. Let $G=(V, E)$ be a graph embedded on the compact orientable surface $S$ and let $C_{1}, \ldots, C_{k}$ be closed curves on $S$. Then there exist closed curves $C_{i 1}, \ldots, C_{i r_{i}} \sim C_{i}$ in $G$ and rationals $\lambda_{i 1}, \ldots, \lambda_{i r_{i}}>0$ for $i=1, \ldots, k$ such that

$$
\begin{aligned}
& \text { (i) } \sum_{j=1}^{r_{i}} \lambda_{i j}=1 \quad(i=1, \ldots, k), \\
& \text { (ii) } \sum_{i=1}^{k} \sum_{j=1}^{r_{1}} \lambda_{i j} \chi^{C_{i j}}(e) \leq 1 \quad(e \in E)
\end{aligned}
$$

if and only if for each closed curve $D$ on $S$ not intersecting $V$

$$
\operatorname{cr}(G, D) \geq \sum_{i=1}^{k} \min \operatorname{cr}\left(C_{i}, D\right) .
$$

Proof. Directly from Theorem 13 , since (46) is equivalent to the all-one vector $(1 ; \mathbf{1})$ belonging to $K$, while $(47)$ is equivalent to $(1 ; \mathbf{1})$ satisfying (40). 
In general, we cannot require the $\lambda_{i j}$ in (46) to be integer, even if we require $G$ to be Eulerian. That is, the analogue of the "integer-packing" theorem, Theorem 5, does not hold. However, if $S$ is the torus the analogue does hold, as was shown in [1]:

THEOREM 15. Let $G=(V, E)$ be an Eulerian graph embedded on the torus $S$ and let $C_{1}, \ldots, C_{k}$ be closed curves on $S$. Then there exist pairwise edge-disjoint closed curves $C_{1}^{\prime} \sim C_{1}, \ldots, C_{k}^{\prime} \sim C_{k}$ in $G$ (such that no $C_{i}^{\prime}$ traverses any edge more than once) if and only if for each closed curve $D$ on $S$ not intersecting $V$ condition (47) is satisfied.

This theorem can be derived from Theorem 14, in a way similar to the derivation of Theorem 5 from the fractional version of Theorem 5 (i.e., Theorem 4).

A consequence of Theorem 14 similar to Theorems 7 and 8 is the following "homotopic flow-cut theorem":

THEOREM 16. Let $G=(V, E)$ be a planar graph embedded in the plane $\mathbf{R}^{2}$ and let $I_{1}, \ldots, I_{p}$ be some of the faces of $G$, including the unbounded face. Let $P_{1}, \ldots, P_{k}$ be curves in $\mathbf{R}^{2} \backslash\left(I_{1} \cup \cdots \cup I_{p}\right)$ with end points on $\mathrm{bd}\left(I_{1} \cup \cdots \cup I_{p}\right)$. Then there exist paths $P_{i 1}, \ldots, P_{i r_{1}} \sim P_{i}$ in $G$ and rationals $\lambda_{i 1}, \ldots, \lambda_{i r_{i}}>0$ for $i=1, \ldots, k$ such that

$$
\begin{array}{ll}
\text { (i) } \sum_{j=1}^{r_{i}} \lambda_{i j}=1 & (i=1, \ldots, k), \\
\text { (ii) } \sum_{i=1}^{k} \sum_{j=1}^{r_{i}} \lambda_{i j} \chi^{P_{i j}}(e) \leq 1 & (e \in E)
\end{array}
$$

if and only if for each curve $D$ in $\mathbf{R}^{2} \backslash\left(I_{1} \cup \cdots \cup I_{p}\right)$ not intersecting $V$ and connecting two points on $\operatorname{bd}\left(I_{1} \cup \cdots \cup I_{p}\right)$ :

$$
\operatorname{cr}(G, D) \geq \sum_{i=1}^{k} \min \operatorname{cr}\left(P_{i}, D\right) .
$$

[Here we use similar terminology and notation as above. A curve is a continuous function $C:[0,1] \rightarrow \mathbf{R}^{2}$, while homotopic requires fixing the end points.]

Proof (SKeTCH). We can reduce this theorem to Theorem 14 by adding for each $i=1, \ldots, k$ a handle connecting the end vertices of $P_{i}$, extending the graph by an edge over this handle (connecting the end points of $P_{i}$ ) and by extending $P_{i}$ to a closed curve over the handle.

We now finally come to showing Theorems 17 and 18 .

THEOREM 17. Let $C_{1}, \ldots, C_{k}$ and $C_{1}^{\prime}, \ldots, C_{k^{\prime}}^{\prime}$ be primitive closed curves on the compact orientable surface $S$. Then the following are equivalent:

(i) $k=k^{\prime}$ and there exists a permutation $\pi$ of $\{1, \ldots, k\}$ so that $C_{\pi(i)}^{\prime} \sim C_{i}$ or $C_{\pi(i)}^{\prime} \sim C_{i}^{-1}$ for each $i=1, \ldots, k$; 
(ii) for each closed curve $D$ on $S$

$$
\sum_{i=1}^{k} \min \operatorname{cr}\left(C_{i}, D\right)=\sum_{i=1}^{k^{\prime}} \min \operatorname{cr}\left(C_{i}^{\prime}, D\right) \text {. }
$$

Proof (Sketch). The implication (i) $\Rightarrow$ (ii) is trivial since $\min \operatorname{cr}\left(C_{i}^{-1}, D\right)$ $=\min \operatorname{cr}(C, D)$. To see the implication (ii) $\Rightarrow$ (i) we may assume that both $C_{1}, \ldots, C_{k}$ and $C_{1}^{\prime}, \ldots, C_{k^{\prime}}^{\prime}$ form minimally crossing collections of closed curves, and that the system $C_{1}, \ldots, C_{k}$ has at least as many crossings as $C_{1}^{\prime}, \ldots, C_{k^{\prime}}^{\prime}$. Let $G=(V, E)$ be the graph made up by $C_{1}^{\prime}, \ldots, C_{k^{\prime}}^{\prime}$. Without loss of generality, each vertex of $G$ has degree 2 or 4 .

Now by (ii), for each closed curve $D$ on $S$ not intersecting $V$

$$
\operatorname{cr}(G, D)=\sum_{i=1}^{k^{\prime}} \operatorname{cr}\left(C_{i}^{\prime}, D\right) \geq \sum_{i=1}^{k^{\prime}} \min \operatorname{cr}\left(C_{i}^{\prime}, D\right)=\sum_{i=1}^{k} \min \operatorname{cr}\left(C_{i}, D\right) .
$$

Hence by Theorem 14 there exist $C_{i j} \sim C_{i}$ and $\lambda_{i j}>0$ satisfying (46).

Now it can be proved that each $C_{i j}$, if it enters a vertex $v$ over an edge $e$, continues over the edge $e^{\prime}$ opposite to $e$ :

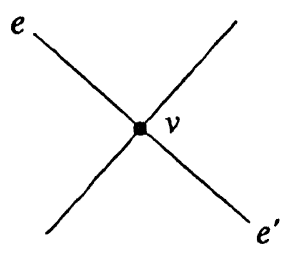

The reason is that $C_{1}, \ldots, C_{k}$ necessarily have at least as many crossings as $C_{1}^{\prime}, \ldots, C_{k^{\prime}}^{\prime}$. Hence the $C_{i j}$ should "use" all crossings of the $C_{i}^{\prime}$-if any $C_{i j}$ makes a turn in $v$, there is not enough room left for crossings of the $C_{i j}$. This intuitive argument can be made precise at the cost of several technicalities-see [8].

It follows that each $C_{i j}$ in fact is one of $C_{1}^{\prime}, \ldots, C_{k^{\prime}}^{\prime}$ and their inverses. As we may assume that the $C_{i j}$ are different, the theorem now follows.

Finally

THEOREM 18. Let $G$ and $G^{\prime}$ be cellularly embedded kernels on $S$ with $\mu_{G}=\mu_{G^{\prime}}$. Then $G^{\prime}$ can be obtained from $G$ by a series of the following operations:

(i) shifting the graph homotopically over $S$;

(ii) taking the (surface) dual graph;

(iii) $\Delta Y$-exchange.

Proof (SKETCH). From $G$ we make an auxiliary graph $H$ as follows. For each edge $e$ of $G$, put a vertex $w_{e}$ of $H$ on the "middle" of $e$. For each 
vertex $v$ of $G$, make a circuit connecting the vertices $w_{e}$ on the edges $e$ of $G$ incident to $v$ :

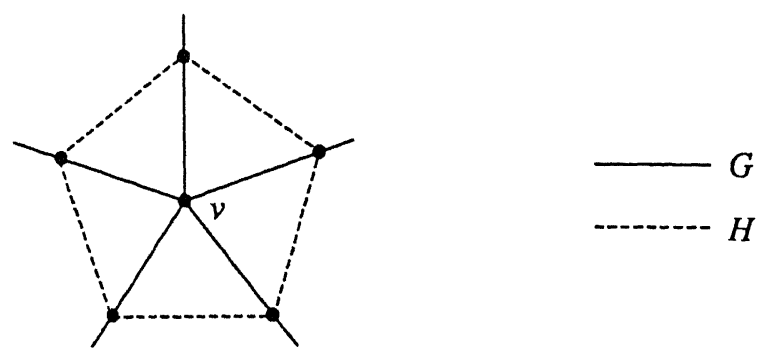

Thus we obtain a 4-regular graph $H$. Note that we can reconstruct $G$ from $H$, up to shifting $G$ and up to duality.

Now deletion and contraction of an edge $e$ of $G$ corresponds to the two ways of opening vertex $w_{e}$ of $H$. Moreover, $\bar{\mu}_{H}=2 \mu_{G}$. Therefore, as $G$ is a kernel, $H$ is tight.

Similarly, we make a tight graph $H^{\prime}$ from $G^{\prime}$. Then

$$
\bar{\mu}_{H^{\prime}}=2 \mu_{G^{\prime}}=2 \mu_{G}=\bar{\mu}_{H} .
$$

Let $C_{1}, \ldots, C_{k}$ and $C_{1}^{\prime}, \ldots, C_{k^{\prime}}^{\prime}$ be the straight decompositions of $H$ and $H^{\prime}$, respectively. By Theorem 10 we have for each closed curve $D$

$$
\sum_{i=1}^{k} \min \operatorname{cr}\left(C_{i}, D\right)=\bar{\mu}_{H}(D)=\bar{\mu}_{H^{\prime}}(D)=\sum_{i=1}^{k^{\prime}} \min \operatorname{cr}\left(C_{i}^{\prime}, D\right) .
$$

So by Theorem 17 we may assume that $k=k^{\prime}$ and that $C_{i} \sim C_{i}^{\prime}$ for $i=1, \ldots, k$.

By Theorem 9 , both $C_{1}, \ldots, C_{k}$ and $C_{1}^{\prime}, \ldots, C_{k}^{\prime}$ are minimally crossing collections of primitive closed curves. It can be shown (using the hyperbolic plane representation of the universal covering surface of $S$ ) that $C_{1}, \ldots, C_{k}$ can be shifted to $C_{1}^{\prime}, \ldots, C_{k}^{\prime}$ keeping the collection minimally crossing throughout the shifting process. In fact $C_{1}, \ldots, C_{k}$ can be transformed to $C_{1}^{\prime}, \ldots, C_{k}^{\prime}$ by a number of "swappings", i.e., replacing

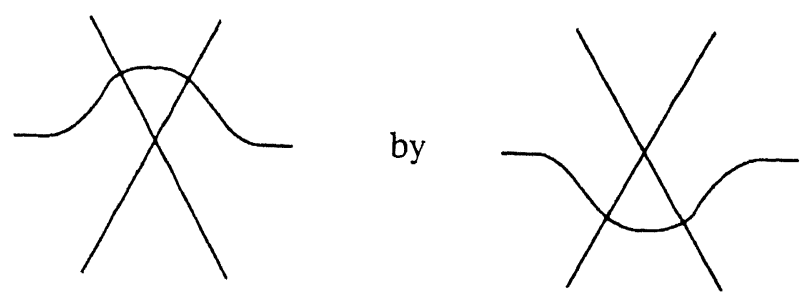

(and by shifting the whole graph $C_{1} \cup \cdots \cup C_{k}$ ).

Any such swapping corresponds to transforming $H$, and hence to transforming $G$. One easily checks that it corresponds to the $\Delta Y$-exchange. Hence $G^{\prime}$ can be obtained from $G$ by the operations (i), (ii), and (iii). 


\section{REFERENCES}

1. A. Frank and A. Schrijver, Disjoint homotopic cycles on the torus, preprint.

2. D. R. Fulkerson, Blocking and anti-blocking pairs of polyhedra, Math. Programming 1 (1971), 168-194.

3. S. Lins, A minimax theorem on circuits in projective graphs, J. Combin. Theory Ser. B 30 (1981), 253-262.

4. H. Okamura, Multicommodity flows in graphs, Discrete Appl. Math. 6 (1983), 55-62.

5. H. Okamura and P. D. Seymour, Multicommodity flows in planar graphs, J. Combin. Theory Ser. B 31 (1981), 75-81.

6. S. Randby, personal communication by N. Robertson (1989).

7. A. Schrijver, Distances and cuts in planar graphs, J. Combin. Theory Ser. B 46 (1989), 46-57.

8. __ Homotopy and crossings of systems of curves on a surface, Linear Algebra Appl. 114 (1989), 157-167.

9. Decomposition of graphs on surfaces and a homotopic circulation theorem, Report OS-R8719, Mathematical Centre, 1987 (to appear in Journal of Combinatorial Theory (B)).

10. The Klein bottle and multicommodity flows, Combinatorica 9 (1989), 375-384.

11. _ . On the uniqueness of kernels, Report OS-R8924, Mathematical Centre, 1989.

Mathematical Centre, Amsterdam, The Netherlands 